\title{
Potencial anti-apoptótico de p38 en cáncer de próstata
}

\author{
M. Ricote Belinchón ${ }^{1}$, F.R. Bethencourt $\operatorname{Codes}^{2}$, I. García-Tuñón Llanio ${ }^{1}$, B. Fraile Láiz ${ }^{1}$, \\ C. Fernández Sáez ${ }^{3}$, P. Aller Tresguerres ${ }^{3}$, J. González-García ${ }^{2}$, R. Vera San Martín ${ }^{1}$, \\ R. Paniagua Gómez-Álvarez ${ }^{1}$, M. Royuela García ${ }^{1}$ \\ ${ }^{1}$ Departamento de Biología Celular y Genética, Universidad de Alcalá. \\ ${ }^{2}$ Departamento de Urología del Hospital Príncipe de Asturias. Alcalá de Henares. \\ ${ }^{3}$ Centro de Investigaciones Biológicas, Consejo Superior de Investigaciones Científicas. Madrid.
}

Actas Urol Esp 2005; 29 (8): 769-776

\section{RESUMEN}

POTENCIAL ANTI-APOPTÓTICO DE P38 EN CÁNCER DE PRÓSTATA

Introducción: En cáncer de próstata, la vía de señalización intracelular de TNF- $\alpha$ parece estar desviada hacia la activación de p38. p38 podría proteger a las células tumorales de la muerte inducida por TNF- $\alpha$. Nos propusimos estudiar el papel que desempeña p38 tanto in vivo (evaluando algunos factores activados por p38 en cáncer de próstata), como in vitro (en las líneas celulares tumorales de próstata LNCaP y PC3, tratadas previamente con TNF- $\alpha$ ).

Material y métodos: Para los estudios in vitro se utilizaron las líneas celulares LNCaP y PC3. Los tratamientos se realizaron con TNF- $\alpha$ (diferentes concentraciones) y el inhibidor específico de p38 (SB203580). El índice apoptótico se evaluó mediante DAPI y citometría de flujo. La activación de p38 se determinó mediante Western blot. Para los estudios in vivo se usaron 15 próstatas normales (PN) y 27 de cáncer (CP) procesadas para inmunohistoquímica y Western blot.

Resultados: In vitro, el aumento de la concentración de TNF- $\alpha$ indujo apoptosis en LNCaP, pero no en PC3. El tratamiento con TNF- $\alpha$ produjo un aumento de la fosforilación de p38 en concentraciones intermedias, mientras que en PC3 no se observaron cambios en la activación. El pretratamiento con SB203580 y TNF- $\alpha$ produjo un aumento significativo de la apoptosis en LNCaP.

In vivo todos los pacientes con PN resultaron positivos para p-Elk-1 y p-ATF-2 (núcleo de células epiteliales). En CP no sólo aumenta la expresión de estos factores, sino que se localizaron tanto en núcleo como en citoplasma.

Conclusión: Nuestros datos in vitro e in vivo sugieren que p38 juega un importante papel en la progresión del cáncer de próstata. Estas observaciones sugieren que tratamientos centrados en el control de la activación de p38 podrían ser efectivos en el tratamiento contra el cáncer de próstata.

Palabras clave: Cáncer de próstata. TNF- $\alpha$. p38. LNCaP. PC3.

\section{ABSTRACT}

ANTI-APOPTOTIC POTENCIAL ROLE OF P38 IN PROSTATE CANCER

Introduction: TNF- $\alpha$ transduction pathway in prostate cancer seems to be diverted towards p38 activation. P38 may protect prostate tumoral cells from TNF- $\alpha$ apoptosis induced. The aim of this study was study the role of p38 in vivo (were evaluated some p38 downstream factors), as well as in vitro (in prostatic tumoral cell lines, LNCaP and PC3, pre-treated with TNF- $\alpha$ ).

Material and methods: Two prostatic tumoral cell lines (LNCaP and PC3) were used in in vitro studies. Two different experiments were made: with TNF- $\alpha$ (several concentrations) and p38 specific inhibitor (SB203580). The apoptotic index were evaluated using DAPI staining and flow cytometry. P38 activation was measured by Western blot analysis. 15 normal samples (NP) and 27 prostate cancer samples (PC) were used in in vivo study, all of them were processed for immunohistochemistry and Western-blot.

Results: In vitro, TNF- $\alpha$ induced apoptosis in LnCap when we increased its concentration but not in PC3. TNF- $\alpha$ stimulation led to increase a time-dependent p38 phosphorylation in two intermediate doses whereas in PC3 not changes were found. In LNCaP after its preincubation with SB203580 and TNF- $\alpha$ treatment showed a significative increasing of apoptosis.

In vivo, all NP samples were found positives to p-Elk-1 and p-ATF-2 (nuclei of epithelial cells). In PC the expression of p-Elk-1 or p-ATF-2 increased and was located in the nucleus and cytoplasm of epithelial cells.

Conclusion: Our data in vitro and in vivo suggest that p38 plays a very important role in prostatic tumour progression. These data suggest that the control activation of p38 might be a possible target to cancer prostate treatment.

Keywords: Prostate cancer. TNF- $\alpha$. p38. LNCaP. PC3. 
$\mathrm{E}$ papel de las citoquinas en el cáncer de próstata es muy poco conocido. En concreto, TNF- $\alpha$ es un miembro vital de la superfamilia del TNF que desempeña un papel muy importante en el sistema inmune, remodelación celular, apoptosis y supervivencia celular ${ }^{1}$. TNF- $\alpha$ desempeña un papel multifuncional y a veces opuesto en la evolución y el tratamiento del cáncer. Lejeune et al. ${ }^{2}$ observaron que la administración local de altas dosis de TNF- $\alpha$ destruían selectivamente los vasos sanguíneos del tumor y por lo tanto su gran poder anticancerígeno en melanoma. Sin embargo, diferentes estudios sugieren que TNF- $\alpha$ endógeno está implicado en el crecimiento de las células tumorales y en las interacciones con el estroma que facilitan la invasión tumoral y el desarrollo de metástasis ${ }^{3,4}$. Uno de los aspectos más importantes de la regulación de la muerte celular son las proteínas activadas por mitógenos (MAPKs) ${ }^{5}$. En mamíferos esta familia de proteínas la componen principalmente tres miembros: JNK, p38 y ERK. Aunque hay múltiples excepciones JNK y p38 se han relacionado con la inducción de apoptosis mientras que ERK con mitogénesis y de manera inversa con apoptosis ${ }^{5}$.

TNF- $\alpha$ induce apoptosis cuando interacciona con su receptor de tipo I (TNFRI), el cual va a unir la molécula adaptadora al dominio de muerte celular (TRADD), la unión de ambas proteínas provoca la activación de toda una cascada de transducción intracelular mediada por fosforilaciones sucesivas (TRAF-2, ASK-1, MEK-4, JNK y AP-1) ${ }^{6}$ (Fig. 1). Pero la unión TNFRI-TRADD también puede desencadenar una señal intracelular que promueve la supervivencia celular y la inhibición de la apoptosis mediante la activación del factor nuclear-kB $(\mathrm{NF}-\mathrm{kB})^{7}$. Además, la vía de señalización TNF- $\alpha$ /AP- 1 cuenta con dos intermediarios que son capaces de activar p38: ASK-1 (que fosforila MEK-6) y MEK-4 (que fosforila directamente p38) ${ }^{8}$. Por esta diversidad en cuanto a las señales que es capaz de inducir TNF- $\alpha$, nos propusimos estudiar las diferentes rutas que promueven la activación de las MAPKs (JNK y p38), así como algunas dianas proteicas (factores de transcripción) in vivo (mediante inmunohistoquímica y Western blot en muestras procedentes de pacientes con cáncer de próstata) e in vitro (en las líneas celulares tumorales de próstata humana LNCaP y PC3).

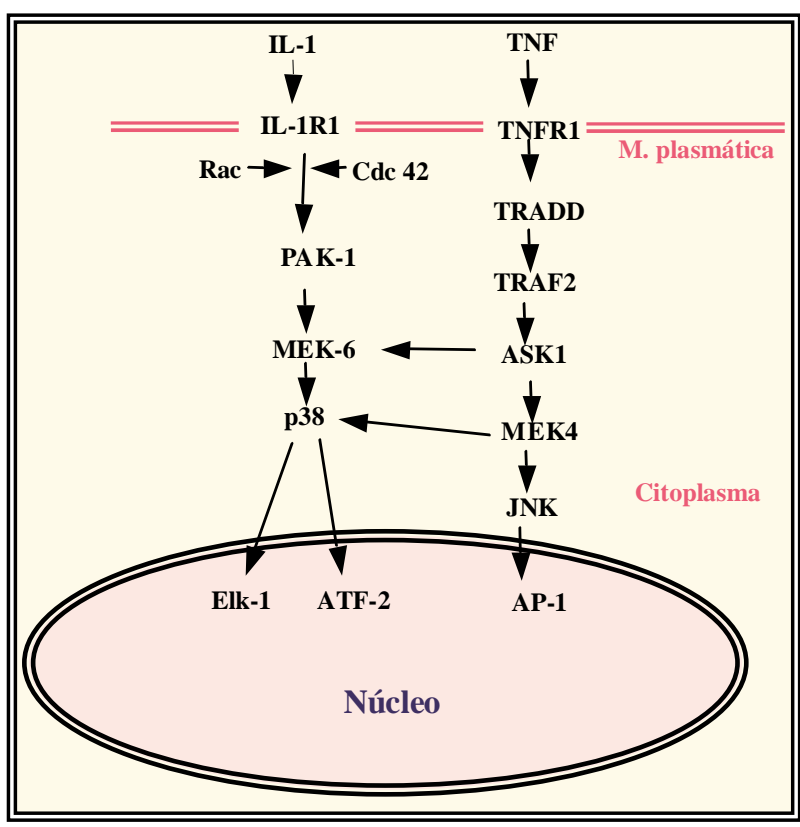

FIGURA 1. Ruta de transducción inducida por TNF- $\alpha$ e IL-1.

\section{MATERIALES Y MÉTODOS}

La experimentación in vitro se realizó con dos líneas celulares de origen humano obtenidas de la empresa ATCC (www.atcc.org).

1. LNCaP: CRL-1740, clon FGC de American Type Collection (ATCC, Rockville, MD). Esta línea celular es sensible a 5 - $\alpha$-dihidrotestosterona, modulando el crecimiento celular y la producción de la fosfatasa ácida prostática.

2. PC3: CRL-1435 de American Type Collection (ATCC, Rockville, MD). Estas células producen una cantidad baja de fosfatasa ácida y presentan actividad testosterona-5- $\alpha$-reductasa. Las células PC3 no responden a andrógenos, glucocorticoides, ni a los factores de crecimiento epidérmico (EGF) o fibroblástico (FGF). Ambas líneas celulares se sometían a controles bimensuales para micoplasma.

Para realizar los estudios in vivo se utilizaron 15 próstatas normales (PN) procedentes de autopsias de varones sin patología reproductiva o endocrina. 27 pacientes que presentaban adenocarcinoma de próstata (CP) con edades comprendidas entre los 54 y 69 años. Cada muestra se dividió en tres porciones: una procesada inmediatamente para inmunohistoquímica y otras dos porciones congeladas en nitrógeno líquido y mantenidas a $-80^{\circ} \mathrm{C}$ para Western blot. Este estudio 
ha sido aprobado por el comité Ético del Hospital y realizado con el consentimiento de los respectivos pacientes.

\section{Cultivos celulares}

Para la realización de los experimentos con LNCaP y PC3, las células se sembraron 24 horas antes de comenzar el experimento para conseguir su adherencia a la placa de cultivo y una confluencia celular del $60 \%$. Los tratamientos con TNF- $\alpha$ se hicieron a diferentes concentraciones (10, 20, 50, 75 y $100 \mathrm{ng} / \mathrm{ml})$. El inhibidor específico de p38, SB203580 procedente de Calbiochem (Darmstadt, Alemania), se preincubó 6 horas antes del tratamiento con TNF- $\alpha$. En cada uno de los experimentos se testó el índice apoptótico mediante dos procedimientos: examen microscópico de la fragmentación de la cromatina teñida con DAPI y cuantificación del contenido de ADN, al que se ha incorporado ioduro de propidio, mediante citometría de flujo.

Tras cada experimento, todas las células tratadas se recogen y son lavadas varias veces con PBS y resuspendidas en este tampón. Para realizar la tinción DAPI, parte de las células recolectadas son extendidas en portaobjetos y se dejan secar, a continuación se fijan en una solución de etanol al 70\% (v/v). Después se incuban durante 20 minutos con 4,6-diamino-2-phenilindol (DAPI) (Serva, Heidelberg, Germany) a temperatura ambiente y se dejan secar los portaobjetos. A continuación se dispone un cubreobjetos sobre las células teñidas utilizando como medio de montaje $10 \%(\mathrm{v} / \mathrm{v})$ PBS, $90 \%$ (v/v) glicerol y 0,1\% $(\mathrm{p} / \mathrm{v})$ de o-fenilendiamina. Las muestras se examinan con microscopio de fluorescencia considerando las células que presentan cromatina fragmentada como apoptóticas. Para medir el contenido de DNA mediante citometría de flujo se toma parte de las células que se recogieron y se resuspenden con RNAasa $(0,5 \mathrm{mg} / \mathrm{ml})$ en PBS incubándolas a temperatura ambiente durante 20 minutos. Se añade Nonidet P-40 al $1 \%$ para permeabilizar las células y finalmente se ponen $5 \mu \mathrm{l}$ de ioduro de Propidio cinco segundos antes de medir las muestras. Para la medición se utiliza un filtro apropiado para detectar la emisión con un pico de $590 \mathrm{~nm}$. Las células hipodiploides (subG1) serán las consideradas como apoptóticas.

\section{Western blot}

Para Western blot, tanto los tejidos como las células fueron homogeneizados en un tampón de extracción (Tris- $\mathrm{HCl} 0,05 \mathrm{M} \mathrm{y} \mathrm{pH}$ : 8) con una mezcla adicional de inhibidores de proteasas (10 mM iodoacetamida, $100 \mathrm{mM}$ phenil-metil sulfónico fluorado, $0,01 \mathrm{mg} / \mathrm{ml}$ inhibidores de la tripsina de soja y $1 \mu \mathrm{l} / \mathrm{ml}$ de leupeptina) junto con Tritón X-100 al 0,5\%. Los homogeneizados se centrifugaron 10 minutos a $10.000 \mathrm{rpm}$, desechando los precipitados. Con el fin de poder comparar los resultados obtenidos en las diferentes muestras, se calculó la concentración de proteína en los distintos sobrenadantes mediante la técnica Bradford. Posteriormente, los sobrenadantes se mezclaron con tampón de muestra (SDS al $10 \%$ en Tris- $\mathrm{HCl} \mathrm{pH} 8$ con glicerol al $50 \%, 2-\beta-$ mercaptoetanol $0,1 \mathrm{mM}$ y azul de bromofenol al $0,1 \%$ ), se calentaron a $100^{\circ} \mathrm{C}$ durante 5 minutos y se cargaron alícuotas de $10 \mathrm{ml}$ en minigeles de SDS-poliacrilamida con un gradiente de concentración del $15 \%$. Las proteínas se transfirieron a membranas de nitrocelulosa. Posteriormente, estas membranas se sumergieron en Blotto (SIGMA) al 5\% en PBS durante 1 hora a temperatura ambiente en agitación. Se incubaron (toda la noche a temperatura ambiente y agitación) con anticuerpo primario diluido en solución de bloqueo (diluida 1+9 en TBS). Las diluciones usadas para los anticuerpos primarios fueron de 1:100 en estudios in vivo (p-p38, p-ATF-2 y p-Elk-1 procedentes de Santa Cruz Biotechnology, Santa Cruz, California, USA) y 1:1000 en estudios in vitro para JNK y p38 en su forma total y fosforilada (procedentes de Cell Signaling Technology, Beverly MA, USA).

Se lavaron tres veces con solución de lavado (5 minutos cada lavado) y se incubaron con el anticuerpo secundario unido a peroxidasa a una dilución 1:4000 en solución de bloqueo (diluida 1+9 en TBS) durante 1 hora a temperatura ambiente con agitación. Tras tres lavados de 10 minutos cada uno se reveló la actividad peroxidasa usando el kit ECL de Amershan.

\section{Inmunohistoquímica}

Los tejidos se fijaron en formaldehido al $10 \%$ en tampón fosfato $0,1 \mathrm{M}$ durante 24 horas, deshidratados y embebidos en parafina. Las seccio- 
nes se procesaron siguiendo el método del ABC. Tras desparafinar, las secciones se hidrataron e incubaron durante 30 minutos en $\mathrm{H}_{2} \mathrm{O}_{2}$ al $0,3 \%$ en metanol para inhibir la actividad de la peroxidasa endógena. Posteriormente se incubaron durante toda la noche a $4^{\circ} \mathrm{C}$ con los anticuerpos primarios monoclonales a una dilución de 1:25 en TBS con BSA al 1\%. Posteriormente las secciones se lavaron y se incubaron con inmunoglobulinas biotiniladas anti-anticuerpo primario. Tras un nuevo lavado en TBS se incubaron con el complejo $\mathrm{ABC}$ y se revelaron con $\mathrm{DAB}$ (diaminobenzidina tetrahidroclorada) (Sigma, Barcelona, España). Finalmente las secciones se deshidrataron y se montaron en DePex (Probus, Barcelona, España).

La especificidad de la técnica se chequeó realizando una serie de controles negativos y positivos. Como negativo, secciones de cada grupo (normal y cáncer) se incubaron con suero preinmune a la misma concentración de inmunoglobulinas usadas para cada anticuerpo primario. Como positivo, se incubaron secciones de piel con los mismos anticuerpos usados en este estudio.

Para cada anticuerpo, se cuantificó histológicamente la intensidad de marcaje obtenido. En cada próstata normal se seleccionaron al azar seis secciones histológicas de cada región (central, intermedia, periférica). Con el objetivo de 40X se midió la intensidad de marcaje (densidad óptica) por unidad de superficie del epitelio mediante un analizador de imagen automático (MIP 4 versión 4,4, Consulting Image, Barcelona, España). Para cada sección positiva se utilizó un control negativo, de tal forma que la densidad óptica del control se restó de la sección marcada. Posteriormente se calculó la media, así como la desviación standard de la media. Los mismos estudios cuantitativos se desarrollaron en PN y CP. La significación estadística se estudió mediante el test de Fisher y Behrens.

\section{RESULTADOS}

\section{ESTUDIOS IN VIVO}

\section{Western blot}

Se observó una única banda para p-p38 y pATF-2 en torno a los 38 y $70 \mathrm{kDa}$ respectivamente en los dos grupos estudiados (PN y CP). En ambos casos el análisis densitométrico de las bandas mostró un incremento en CP frente a PN. p-Elk-1 (66 kDa) se detectó en los dos grupos aunque en CP apareció otra banda de $46 \mathrm{kDa}$. La densidad óptica de la banda de $66 \mathrm{kDa}$ fue mayor en CP. En CP la banda detectada en torno a los $46 \mathrm{kDa}$ mostró una densidad óptica menor a la de 66 kDa (Fig. 2).

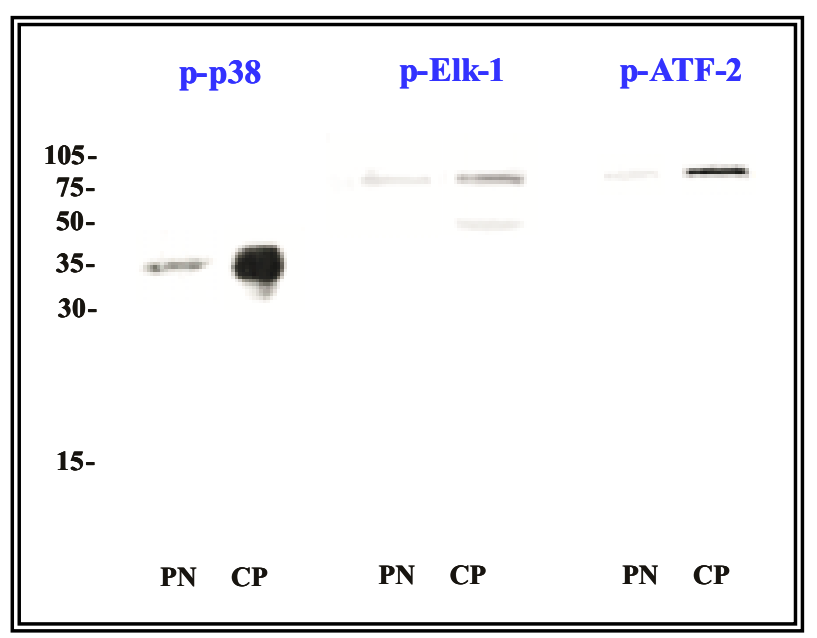

FIGURA 2. Western blot para p-p38, p-Elk y p-ATF-2 en próstata normal (PN) y cáncer de próstata (CP).

\section{Inmunohistoquímica}

El inmunomarcaje para p-p38 en PN apareció en el núcleo de células epiteliales (18\%). En CP (89\%) el porcentaje de muestras con marcaje epitelial aumentó significativamente siendo su localización tanto nuclear como citoplasmática (Fig. $3 \mathrm{~A})$.

p-Elk-1 se observó en el núcleo de las células epiteliales basales en el 100\% de las muestras de PN (Fig. 3B). En CP (Fig. 3C) se localizó en el citoplasma $(51,85 \%$ de las muestras), núcleo $(18,5 \%)$ o tanto en núcleo como citoplasma $(29,6 \%)$. Tras realizar el análisis estadístico se observó un incremento estadísticamente significativo desde $\mathrm{PN}$ a CP.

Para p-ATF-2 el 100\% de las muestras de PN fueron positivas, localizándose en el núcleo de las células epiteliales (Fig. 3D). Un 25,9\% de las muestras de CP mostraron localización nuclear y un 59.2\% localización citoplasmática (Fig. 3E). Las densidades ópticas mostraron un aumento de señal, estadísticamente significativo, que se correspondía con el aumento de la malignidad. 


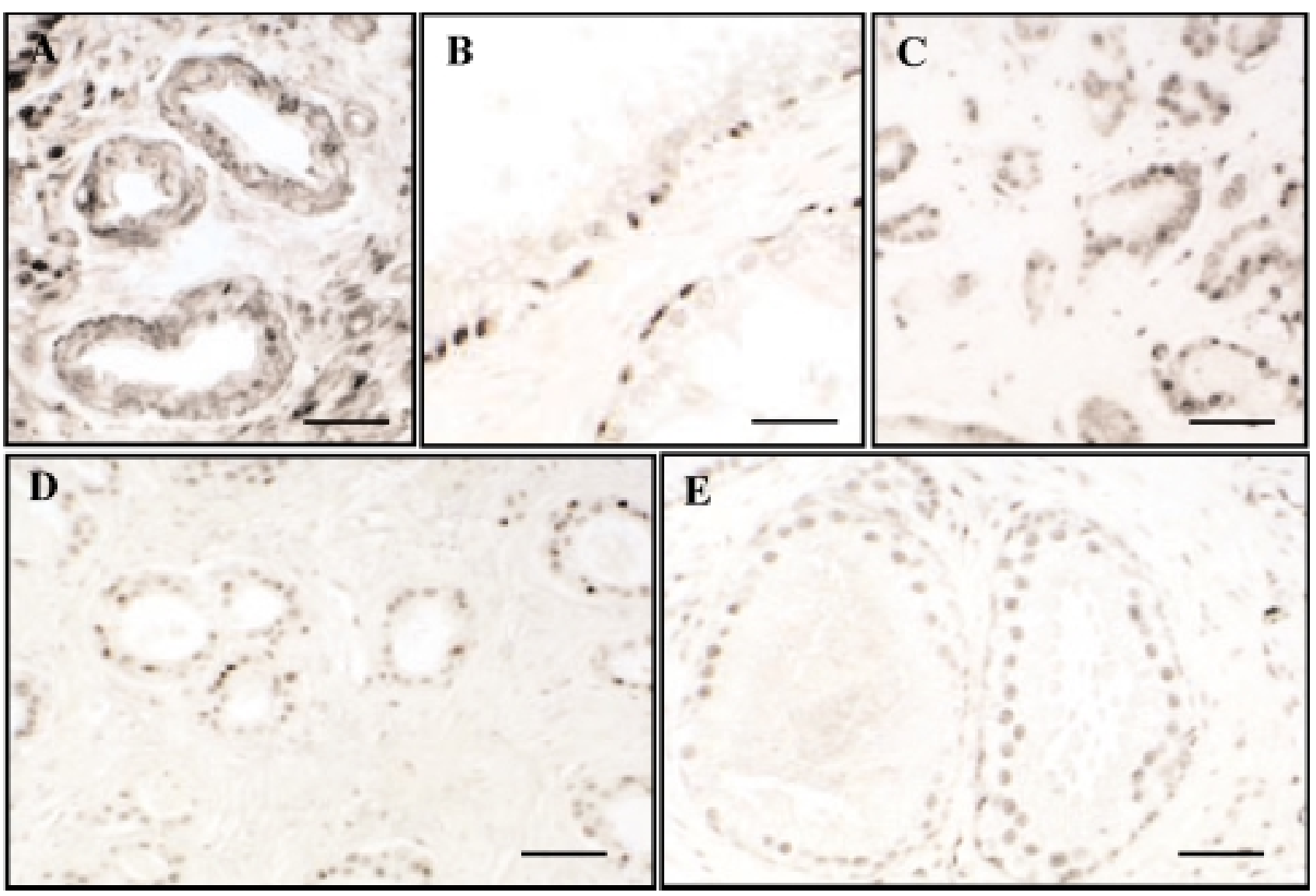

FIGURA 3. p-p38 se localizó en el citoplasma y en algunos núcleos en CP (A). p-Elk-1 apareció en el núcleo de células epiteliales basales de PN (B) y citoplásmico en CP (C). p-ATF-2 se observó en el núcleo de las céllulas epiteliales en PN (D) y CP (E). Barra: $15 \mu \mathrm{m}$.

\section{Estudios in vitro}

Mediante la tinción DAPI se calculó el porcentaje de células apoptóticas después del tratamiento con TNF- $\alpha(10,20,50,75,100 \mathrm{ng} / \mathrm{ml})$ tras 72 horas de incubación (Fig. 4). En las LNCaP el índice apoptótico aumentó con la con- centración de TNF- $\alpha$. En PC3 no se observaron variaciones significativas con las diferentes concentraciones. La distribución celular determinada por incorporación de Ioduro de propidio y medida por citometría de flujo en los cultivos de LNCaP sometidos al mismo tratamiento, mostró

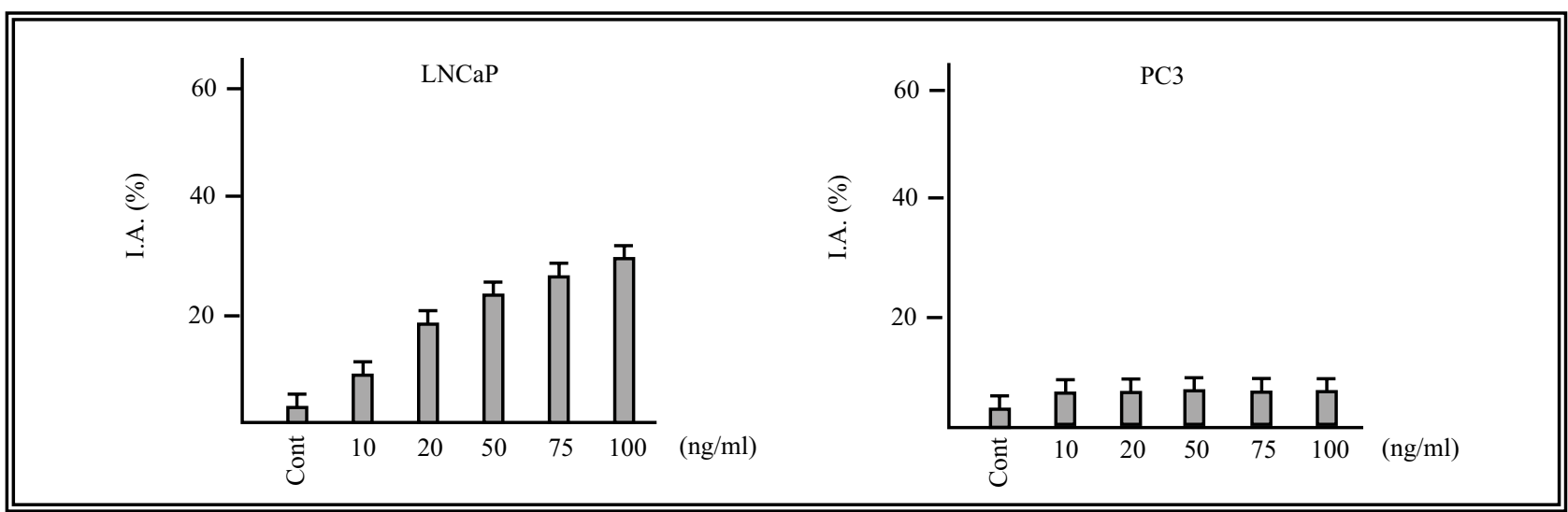

FIGURA 4. Índice apoptótico para LNCaPy PC3 tras el tratamiento con TNF-a a diferentes concentraciones durante 72 horas. I.A. (\%) indice apaptótico expresado en porcentajes. 
un incremento de la porción de células en la fase sub-G $G_{1}$ (Ap) paralelo al aumento de concentración de esta citoquina. Por el contrario, en las células PC3 la proporción de células en fase sub$\mathrm{G}_{1}$ no varió significativamente, por lo que TNF- $\alpha$ no induciría apoptosis en PC3.

Tras el Inmunoblot (Fig. 5) de los diferentes extractos de las células tratadas con TNF- $\alpha$ en concentraciones crecientes $(10,20,50,75,100$ $\mathrm{ng} / \mathrm{ml}$ ) durante 72 horas, se cuantificó la densidad óptica de JNK (total) y p-JNK (fosforilada), observándose en LNCaP un incremento de la proteína fosforilada en las tres últimas dosis administradas (50, 75, $100 \mathrm{ng} / \mathrm{ml})$. El Inmunoblot para p38 (total) y p-p38 (fosforilado) en las mismas condiciones experimentales reveló un aumento de p-p38 a las dosis intermedias en LNCaP (20 y $50 \mathrm{ng} / \mathrm{ml})$. En el caso de PC3 no se observaron diferencias para ninguna de las MAPKs. Todas las diferencias de activación se verificaron mediante la medida de las densidades ópticas.

Para estudiar la posible actuación de p38 se inhibió selectivamente esta proteína y se observó en que medida se alteraba la viabilidad celular (Fig. 6). Las dosis elegidas para realizar estos experimentos fueron de 50 y $75 \mathrm{ng} / \mathrm{ml}$, ya que en estas concentraciones se había identificado una respuesta "regulable" de la acción de TNF- $\alpha$. En este caso se encontró que se producía una inducción muy significativa de la apoptosis. La presencia de este inhibidor llegó a duplicar el índice apoptótico que se encontró cuando sólo había en

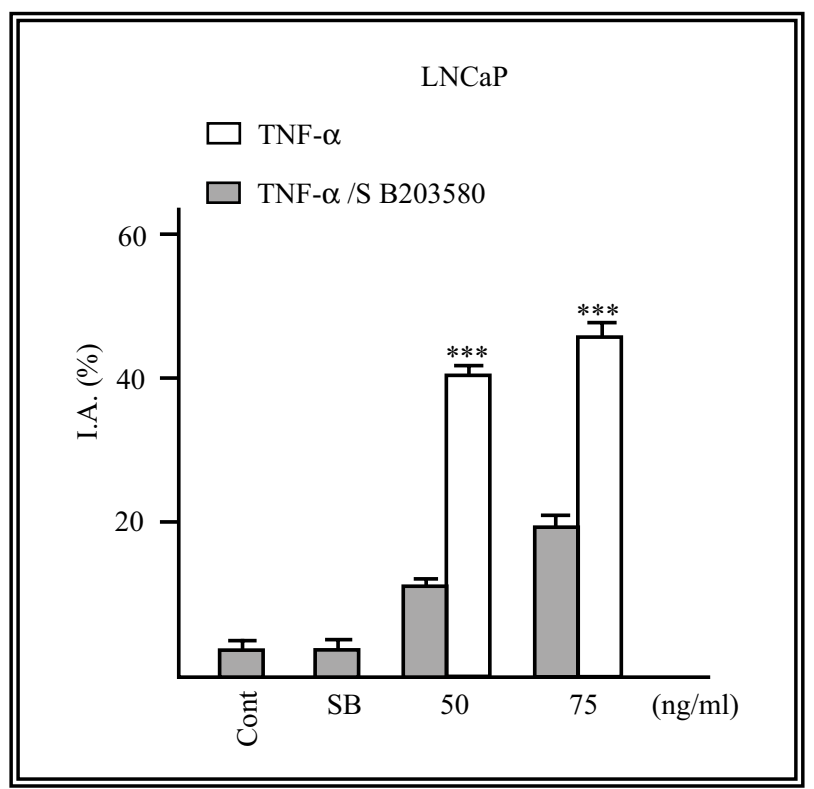

FIGURA 6. Índice apoptótico para LNCaP tras el tratamiento con TNF- $\alpha$ a diferentes concentraciones durante 72 horas. ${ }^{* * *} p<0,001$.

el medio TNF- $\alpha$. Mediante citometría de flujo los datos que encontramos revelaban también un aumento significativo de apoptosis, en los casos que se había pre-tratado con inhibidor.

\section{DISCUSIÓN}

Con anterioridad nuestro grupo estudió en profundidad la vía de señalización TNF- $\alpha$ / JNKAP- $1^{9}$ en próstata humana tanto normal como con cáncer, concluyendo que en la patología maligna esta ruta se encuentra desactivada, desviada o inhibida en alguno de sus intermediarios

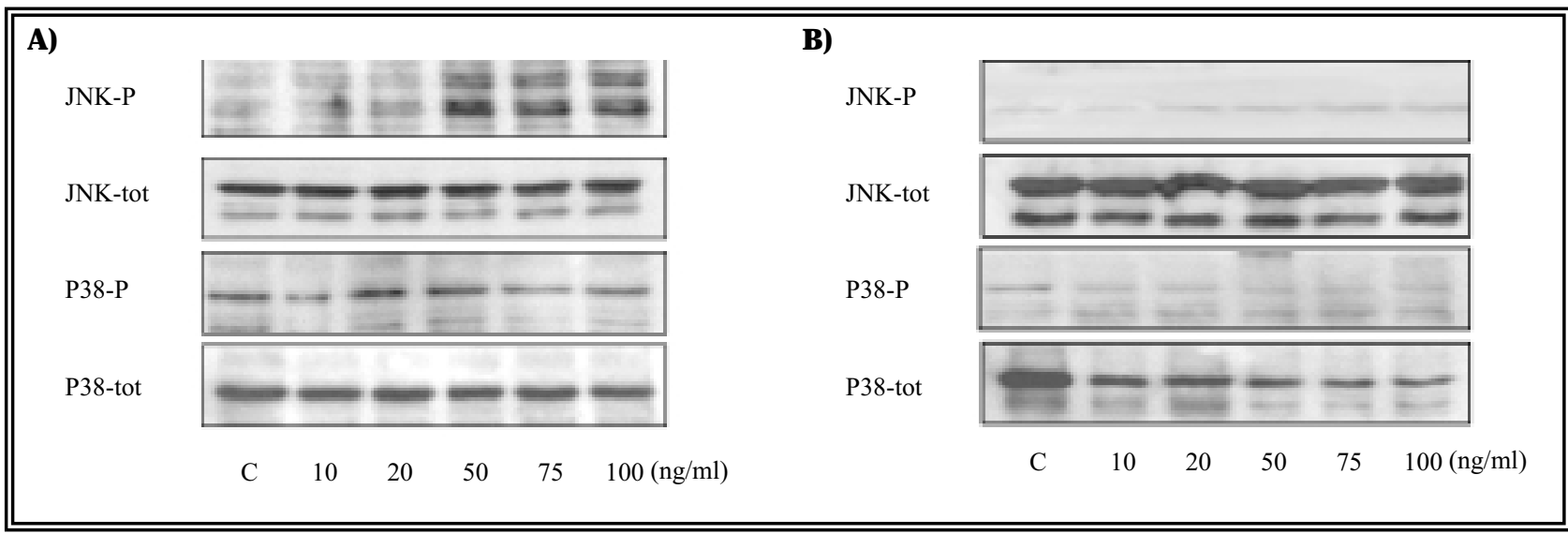

FIGURA 5. Western blot para p-JNK (fosforilada) y tot-JNK (total), p-p38 (fosforilada) y tot-p38 (total) en LNCaP (A) y PC3 (B) a las diferentes concentraciones de TNF- $\alpha$. 
pues la señal disminuía en los últimos componentes de esta ruta. Algunos de los intermediarios de la ruta están desviados hacia la activación de p38, proteína que además puede ser activada por IL- ${ }^{10}$. Nuestro grupo también describió una elevada inmunoexpresión de IL- $1 \alpha$ y del receptor IL-1RI ${ }^{11}$. Por ello, los resultados de este estudio sobre p38 y los factores de transcripción que ésta activa nos pueden dar información sobre el papel de TNF- $\alpha$ e IL- 1 en el control de la proliferación/ apoptosis en próstata humana.

En PN la inmunoexpresión para p-ATF-2 y pElk-1 se observó en los núcleos de las células epiteliales. Proteínas intermediarias en la activación de p38 por IL-1, como PAK-1, fueron negativas (datos no mostrados). Esto sugiere que la activación de ambos factores de transcripción y p38 no está inducida por IL-1 sino por la vía TNF- $\alpha$ / MEK-4/p38, ya que la presencia tanto de p38 ${ }^{12}$ como de MEK- $4^{9}$ se describió en las muestras de PN de los mismos varones de este estudio.

En CP se observó una elevada inmunoexpresión para p38 lo que sugiere que esta proteína podría estar hiperactivada ya sea por la presencia de IL-1 o por la desviación de la ruta TNF- $\alpha$ / MEK-4. En CP los factores de transcripción $p$ ATF-2 y p-Elk-1 cambian su localización desde al núcleo al citoplasma en un número elevado de casos. Este hecho podría estar relacionado con un cambio en la función biológica que desempeñan en la patología cancerígena. En células de mamíferos, p38 endógeno se localiza en el núcleo pero puede ser exportado al citoplasma tras su fosforilación (forma activa) ${ }^{12}$. En el núcleo p38 fosforila Elk-1 y ATF-2 ${ }^{13}$. Nuestro estudio realizado mediante Western blot revela dos bandas para p-Elk-1 (en CP) en torno a los 66 y $46 \mathrm{kDa}$ de peso molecular. Similares resultados fueron descritos por Vanhouotte et al. ${ }^{14}$ en neuronas, quienes postularon que la banda de $46 \mathrm{kDa}$ es una nueva isoforma de Elk-1 (s-Elk-1) que podría corresponder a una isoforma truncada de Elk-1 que ha perdido los 54 aminoácidos responsables de la unión con el DNA. s-Elk-1 se acumula en el núcleo compitiendo con Elk-1, y promoviendo la diferenciación celular ${ }^{14}$.

p-ATF-2 también cambia su localización en algunos pacientes de CP, aunque sólo se encontró una banda en el análisis mediante Western blot. Hasta el momento no se conoce el sentido de la localización citoplasmática. La elevada inmunoexpresión encontrada tras el análisis de p38 y los dos factores de transcripción sugieren que p38 puede jugar un papel muy importante en CP. Sin embargo, estos datos serían insuficientes para conocer si p38 está favoreciendo apoptosis o supervivencia/ proliferación celular, así como la actuación de TNF- $\alpha$. Por ello nos propusimos realizar experimentos in vitro con células tumorales humanas (LNCaP y PC3). Ambas líneas celulares son positivas para TNFRI (datos no mostrados). En LNCaP se observó desarrollo de apoptosis dependiente de TNF- $\alpha$ : el aumento de concentración de la citoquina hace aumentar el indice apoptótico. Resultados similares fueron descritos por Kimura et al. ${ }^{15}$. En la línea PC3 no se encontró ninguna alteración en el índice apoptótico con este tratamiento. Esta resistencia de la línea PC3 a TNF- $\alpha$ también ha sido descrita por Sintich et al. ${ }^{16}$. Mediante Western blot también se analizaron JNK y p38 en ambas líneas celulares, encontrándose una respuesta positiva en el caso de LNCaP pero no en PC3. Estas variaciones involucran a estas proteínas en el mecanismo de actuación de TNF- $\alpha$ en cáncer. Para conocer en que sentido actuaba p38 se inhibió selectivamente dicha proteína mediante un inhibidor farmacológico (SB203580) que anularía su posible función tras el tratamiento con TNF- $\alpha$. En este experimento se encontró un desarrollo muy elevado de la apoptosis, duplicando el valor encontrado cuando no se inhibía p38. Este dato sugiere que p38 bajo la estimulación celular con TNF- $\alpha$ tiene un papel protector contra la apoptosis. Este papel protector ha sido sugerido por otros autores sobre las líneas celulares L929 y NIH3T3 ${ }^{17}$. Además, experimentos en los que se han deleccionado los genes para TNF- $\alpha$ y el receptor TNFRI resultaron resistentes al desarrollo de cáncer de piel $^{18,19}$. En otros casos como los desordenes linfoproliferativos de células $\mathrm{B}^{20}$ y en la línea coriocarcinoma humana $\mathrm{JAR}^{21}$, p38 aparece involucrado en oncogénesis en tumores con alta capacidad invasiva.

Teniendo en cuenta estos resultados que sugieren que p38 esta hiperactivada en CP así como los resultados in vitro, que relacionan directamente p38 con la resistencia a apoptosis tras la 
estimulación con TNF- $\alpha$, se sugiere que la inhibición selectiva de p38 mediante fármacos específicos podría representar una nueva estrategia terapéutica para el tratamiento del cáncer de próstata en estadios dependientes de andrógenos.

Agradecimientos. Este trabajo ha sido subvencionado por el Fondo de Investigaciones Sanitarias de la Seguridad Social (PI020383).

\section{REFERENCIAS}

1. Szlosarek PW, Balkwill FR. Tumour necrosis factor alpha: a potential target for the therapy of solid tumours. Oncology 2003;4:565-573.

2. Lejeune FJ, Ruegg C, Lienard D. Clinical applications of TNF-alpha in cancer. Curr Opinion Immunol 1998;10:573580.

3. Beutler B. The role of tumor necrosis factor in health and disease. J Rheumatol 1999;26:16-21.

4. Kollias G, Douni E, Kassiotis G, Kontoyiannis D. On the role of tumor necrosis factor and receptors in models of multiorgan failure, rheumatoid arthritis, multiple sclerosis and inflammatory bowel disease. Immunol Rev 1999;169: 175-194.

5. Cross TG, Scheel-Toellner D, Henriquez NV, Deacon E, Salmon M, Lord JM. Serine/threonine protein kinases and apoptosis. Exp Cell Res 2002;256:34-41.

6. Ichijo H, Nishida E, Irie K, Dijke P, Saitoh M, Moriguchi T, Takagi M, Matsumoto K, Miyazono K and Gotoh Y. Induction of apoptosis by ASK-1, a mammalian MAPKKK that activates SAPK/JNK and p38 signaling pathways. Science 1997;275:90-94.

7. Campbell J, Ciesielski CJ, Hunt AE, Horwood NJ, Beech JT, Hayes LA, Denys A, Feldmann M, Brennan FM, Foxwell BM. A novel mechanism for TNF-alpha regulation by p38 MAPK: involvement of NF-kappa B with implications for therapy in rheumatoid arthritis. J Immunol 2004;173:6928-6937.

8. Stein B, Brady H, Yang MX, Young DB, Barbosa MS. Cloning and characterization of MEK-6, a novel member of the mitogen-activated protein kinase kinase cascade. J Biol Chem 1996; 271: 11427-11433.

8. Ricote M, Royuela M, García-Tuñón I, Bethencourt FR, Paniagua R, Fraile B. Pro-apoptotic tumor necrosis factoralpha transduction pathway in normal prostate, benign prostatic hyperplasia and prostatic carcinoma. J Urol 2003; 170:787-790.

10. Raingeaud J, Gupta S, Rogers J S, Dickens M., Han J, Ulevitch RJ. Davis R.J. Proinflammatory cytokines and environmental stress cause p38 mitogen activated protein kinase activation by dual phosphorylation on tyrosine and threonine. J Biol Chem 1995;270:7420-26.

11. Ricote M, Garcia-Tunon I, Bethencourt FR, Fraile B, Paniagua R, Royuela.. Interleukin-1 (IL-1alpha and IL1beta) and its receptors (IL-1RI, IL-1RII, and IL-1Ra) in prostate carcinoma. Cancer 2004;100:1388-1396.
12. Royuela M, Arenas MI, Bethencourt FR, Sanchez Chapado M, Fraile B, Paniagua R. Regulation of Proliferation/ Apoptosis equilibrium by mitogen-activated protein kinases in Normal, Hyperplastic, and carcinomatous human prostate. Human Pahthol 2000; 33:299-306.

13. Raingeaud J, Whitmarsh AJ, Barrett T, Derijard B, Davis RJ. MKK3- and MKK6-regulated gene expression is mediated by the p38 mitogen-activated protein kinase signal transduction pathway. Mol Cell Biol 1996;16:12471255.

14. Vanhoutte P, Nissen JL, Brugg B, Gaspera BD, Besson MJ, Hipskind RA, Caboche J. Opposing roles of Elk-1 and its brain-specific isoform, short Elk-1, in nerve growth factorinduced PC12 differentiation. J Biol Chem 2001;276:51895196.

15. Kimura K, Bowen C, Spiegel S, Gelmann EP. Tumor necrosis factor-alpha sensitizes prostate cancer cells to gammairradiation-induced apoptosis. Cancer Res 1999; 59:16061614.

16. Sintich SM, Steinberg J, Kozlowski JM, Lee C, Pruden S, Sayeed S, Sensibar JA. Cytotoxic sensitivity to tumor necrosis factor-alpha in PC3 and LNCaP prostatic cancer cells is regulated by extracellular levels of SGP-2 (clusterin). Prostate. 1999;39:87-93.

17. Lüschen S, Scherer, Ussat S, Ungenfroren H, Adam-Klages $\mathrm{S}$. Inhibition of p38 mitogen-activated protein kinase reduces TNF-induced activation of NF-kB, elicits caspase activity, and enhances cytotoxicity. Exp Cell Res 2004;293: 196-206.

18. Moore RJ, Owens DM, Stamp G, Arnott C, Burke F, East N, Holdsworth H, Turner L, Rollins B, Pasparakis M, Kollias G, Balkwill F. Mice deficient in tumor necrosis factor-alpha are resistant to skin carcinogenesis. Nat Med 1999;5:828-831.

19. Suganuma M, Okabe S, Marino MW, Sakai A, Sueoka E, Fujiki H. Essential role of tumor necrosis factor alpha (TNF-alpha) in tumor promotion as revealed by TNF-alphadeficient mice. Cancer Res 1999;59:4516-4518.

20. Zhang S, Han J, Sells MA, Chernorff J, Knaus UG, Ulevitch RJ, Bokoch GM. Rho family GTPases regulate p38 mitogenactivated protein kinase through the downstream mediator PAK1. J Biol Chem 1995;270:23934-23936.

21. Ogasawara T, Yasuyama M Kawauchi K. Constitutive activation of extracellular signal-regulated kinase and p38 mitogen-activated protein kinase in B-cell lymphoproliferative disorders. Int. J. Hematol 2003;77:364-370.

Dra. M. Royuela

Universidad de Alcalá de Henares

Departamento de Biología y Genética

Ctra. de Madrid-Barcelona km. 33600

28871 Alcalá de Henares (Madrid)

e-mail: mar.royuela@uah.es

(Trabajo recibido el 15 de junio 2005) 